\title{
COMMUTING ELEMENTS, SIMPLICIAL SPACES AND FILTRATIONS OF CLASSIFYING SPACES
}

\author{
ALEJANDRO ADEM*, FREDERICK R. COHEN**, AND ENRIQUE TORRES GIESE \\ Abstract. Let $G$ denote a topological group. In this article the descending central series \\ of free groups are used to construct simplicial spaces of homomorphisms with geometric \\ realizations $B(q, G)$ that provide a filtration of the classifying space $B G$. In particular this \\ setting gives rise to a single space constructed out of all the spaces of ordered commuting \\ $n$-tuples of elements in $G$. Basic properties of these constructions are discussed, including \\ the homotopy type and cohomology when the group $G$ is either a finite group or a compact \\ connected Lie group. For a finite group the construction gives rise to a covering space with \\ monodromy related to a delicate result in group theory equivalent to the odd-order theorem \\ of Feit-Thompson. The techniques here also yield a counting formula for the cardinality \\ of $\operatorname{Hom}(\pi, G)$ where $\pi$ is any descending central series quotient of a finitely generated free \\ group. Another application is the determination of the structure of the spaces $B(2, G)$ \\ obtained from commuting $n$-tuples in $G$ for finite groups such that the centralizer of every \\ non-central element is abelian (known as transitively commutative groups), which played a \\ key role in work by Suzuki on the structure of finite simple groups.
}

\section{Contents}

1. Introduction

2. Simplicial Spaces of Homomorphisms

3. Further Properties of $\operatorname{Hom}\left(\mathcal{F}_{n} / \Gamma^{r}, G\right), E(q, G)$ and $B(q, G)$

4. Homotopy Properties of $B(q, G)$ for $G$ a Finite Group

5. Stable Splittings and a Counting Formula for Homomorphisms

6. The Spaces $B(q, G)$ for Connected Lie Groups

7. The Homology of $B(q, G)$ when $G$ is Finite, and the Feit-Thompson Theorem

8. $B(2, G)$ for Transitively Commutative Groups

\section{INTRODUCTION}

Let $G$ denote a topological group. The classifying space $B G$ plays a central role in algebraic topology with important applications to bundle theory and cohomology of groups. In this

Date: May 29, 2018.

*Partially supported by NSERC.

** Partially supported by DARPA and the NSF. 
paper a filtration of $B G$ is introduced by using the descending central series of the free groups. A basic feature is that if $\mathcal{F}_{n}$ is the free group on $n$ generators with $\Gamma^{q}$ the $q$-th stage of its descending central series, then the spaces of homomorphisms $\operatorname{Hom}\left(\mathcal{F}_{n} / \Gamma^{q}, G\right)$ can be assembled to form simplicial spaces with geometric realizations $B(q, G)$ which filter the usual classifying space $B G$. In other words there are inclusions

$$
B(2, G) \subset B(3, G) \subset \cdots \subset B(q, G) \subset B(q+1, G) \subset \cdots \subset B(\infty, G)=B G
$$

where each term is constructed from the simplicial spaces associated to terms in the descending central series of the free group. This naturally gives rise to a functorial construction on topological groups $G \mapsto B(q, G)$. In fact the construction provided here affords a principal $G$-bundle $E(q, G) \rightarrow B(q, G)$ which fits into a commutative diagram, namely there are natural morphisms of principal $G$-bundles

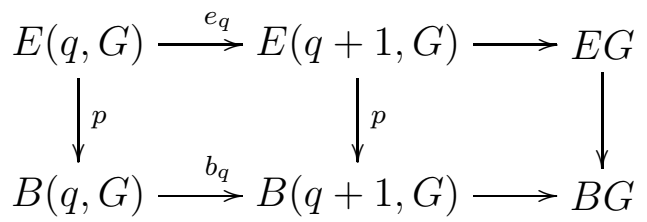

and the maps $e_{q}, b_{q}$ yield a natural filtration of subspaces for $E G$ and $B G$.

When $G$ is a finite group the spaces developed here give finite, regular covering spaces with structure group $G$. General properties arise from features of the monodromy for these covers; one striking fact about the structure is that an elementary property of this natural monodromy is equivalent (see Proposition 7.2) to the Feit-Thompson Theorem about solvability for groups of odd order [10]. The structure of this monodromy will be developed more fully elsewhere, but is addressed here in the case of finite transitively commutative groups, which in the nonabelian case are characterized by the property that all non-central elements have abelian centralizers. These groups were shown to be solvable by Suzuki 26 , when they have odd order, a seminal result that played a key role in the subsequent proof of the Feit-Thompson Theorem (see the survey paper [24], pages 324-325 for an explanation of this).

It turns out that the spaces $B(q, G)$ have many interesting features which are explored in this article; the goal is to introduce these objects and describe both their similarities and differences when compared to standard classifying spaces, connecting them whenever possible to basic properties of spaces of homomorphisms. This will involve different aspects of homotopy theory, group cohomology and group theory. The main focus will be to illustrate these features in basic examples such as finite groups and connected Lie groups.

The paper is organized as follows. Section 2 gives proofs of the simplicial structure of certain spaces of homomorphisms as well as the construction of $B(q, G)$ as the geometric realization of a simplicial space. Section 3 gives general homological, bundle theoretic and geometric properties of the spaces $E(q, G)$ and $B(q, G)$. Section 4 provides a verification that $B(q, G)$ is a natural colimit which is weakly equivalent to a more tractable homotopy colimit. This in turn maps to the classifying space of the colimit of all subgroups in $G$ of nilpotence 
class less than $q$, with fibre a finite dimensional, possibly contractible, complex (Theorem 4.4). In Section 5 a natural stable splitting for certain spaces of homomorphisms is stated, which follows from [2] and [3] under appropriate conditions. In the special case of a finite group $G$, these stable decompositions count the cardinality of $\operatorname{Hom}\left(\mathcal{F}_{n} / \Gamma^{q}, G\right.$ ) (Corollary 5.4). The subject of Section 6 is $B(q, G)$ for a connected Lie groups $G$. For $G$ compact this section gives a computation of the rational cohomology of $B(2, G)$ (Theorem 6.1). It is also shown that the loop space of $B(q, G)$ admits a natural, non-trivial product decomposition $G \times$ $\Omega E(q, G) \simeq \Omega B(q, G)$ (Theorem 6.3). On the other hand, in case $G$ is finite, the homological structure of $B(q, G)$ arises as a natural sub-chain complex of the classical algebraic bar construction. That connection is developed in Section 7 . In addition, the first homology group of $B(q, G)$ as well as the monodromy of the finite cover $E(q, G) \rightarrow B(q, G)$ have a close connection to the Feit-Thompson odd-order theorem, and it is described here (Proposition 7.2). This naturally connects to Section 8 of this paper, where the structure of $E(2, G) \rightarrow$ $B(2, G)$ for transitively commutative groups is described. As has been mentioned above, these groups are key examples in finite group theory. Explicit descriptions are given for these spaces using an action on a tree (Proposition 8.8).

The third author would like to thank Fred Cohen for his hospitality and support given during a stay at the University of Rochester in Spring 2008.

\section{Simplicial Spaces of Homomorphisms}

The goal of this section is to define a family of simplicial spaces assembled using spaces of homomorphisms. In fact, this family will yield a filtration of the bar construction of the classifying space of a group and it will be parametrized by the lower central series of the free groups.

Definition 2.1. Let $Q$ be a group, define a chain of subgroups $\Gamma^{r}(Q)$ inductively: $\Gamma^{1}(Q)=$ $Q ; \Gamma^{i+1}(Q)=\left[\Gamma^{i}(Q), Q\right]$. By convention, $\Gamma^{\infty}(Q)=\{1\}$. The descending central series of $Q$ is the normal series $\cdots \subset \Gamma^{i+1}(Q) \subset \Gamma^{i}(Q) \subset \cdots \subset \Gamma^{2}(Q) \subset \Gamma^{1}(Q)=Q$.

A discrete group $Q$ is said to be nilpotent if there is some integer $m$ such that $\Gamma^{m+1}(Q)=$ $\{1\}$. The least such integer $m$ is called the nilpotency class of $Q$. For example every finite $p$-group is nilpotent.

When the context is clear $\Gamma^{r}$ will be used to denote these normal subgroups of $Q$. Let $\mathcal{F}_{n}=\mathcal{F}\left[r_{0}, r_{1}, \cdots, r_{n-1}\right]$ denote the free group on $n$-letters. Fix an integer $q$ and let $\Gamma^{q}$ denote the $q$-stage of the descending central series for $\mathcal{F}_{n}$, as above.

Let $G$ denote a topological group; in this paper it will be assumed throughout that: (i) $G$ is locally compact as well as Hausdorff; and that (ii) $1 \in G$ is a non-degenerate basepoint. Consider the set $\operatorname{Hom}\left(\mathcal{F}_{n} / \Gamma^{q}, G\right)$ of homomorphisms $f: \mathcal{F}_{n} \rightarrow G$ which descend to homomorphisms $\bar{f}: \mathcal{F}_{n} / \Gamma^{q} \rightarrow G$, i.e. such that $f\left(\Gamma^{q}\right)=1$. An element of $\operatorname{Hom}\left(\mathcal{F}_{n} / \Gamma^{q}, G\right)$ is specified by functions from the set $\left\{r_{0}, r_{1}, \cdots, r_{n-1}\right\}$ to $G$. Thus an element in $\operatorname{Hom}\left(\mathcal{F}_{n} / \Gamma^{q}, G\right)$ is identified as an ordered $n$-tuple $\left(x_{0}, x_{1}, \cdots, x_{n-1}\right)$ with $f\left(r_{i}\right)=x_{i}$ and subject to $f\left(\Gamma^{q}\right)=1$. 
The space $\operatorname{Hom}\left(\mathcal{F}_{n} / \Gamma^{q}, G\right)$ is topologized as a subspace of $G^{n}$ with the naturally inherited topology for general $G$.

Two simplicial spaces associated to these homomorphisms will now be introduced.

Definition 2.2. Let

$$
E_{n}(q, G)=G \times \operatorname{Hom}\left(\mathcal{F}_{n} / \Gamma^{q}, G\right) \subset G^{n+1},
$$

and define $d_{i}: E_{n}(q, G) \rightarrow E_{n-1}(q, G)$ for $0 \leq i \leq n$ and $s_{j}: E_{n}(q, G) \rightarrow E_{n+1}(q, G)$, for $0 \leq j \leq n$, given by

$$
d_{i}\left(g_{0}, \ldots, g_{n}\right)=\left\{\begin{array}{lr}
\left(g_{0}, \ldots, g_{i} \cdot g_{i+1}, \ldots, g_{n}\right) & 0 \leq i<n \\
\left(g_{0}, \ldots, g_{n-1}\right) & i=n
\end{array}\right.
$$

and $s_{j}\left(g_{0}, \ldots, g_{n}\right)=\left(g_{0}, \ldots, g_{i}, 1, g_{i+1}, \ldots, g_{n}\right)$ for $0 \leq j \leq n$.

Definition 2.3. Similarly, let $B_{n}(q, G)=\operatorname{Hom}\left(\mathcal{F}_{n} / \Gamma^{q}, G\right)$ with maps $d_{i}$ and $s_{j}$ defined in the same way, except that the first coordinate $g_{0}$ is omitted and the map $d_{0}$ takes the form $d_{0}\left(g_{1}, \ldots, g_{n}\right)=\left(g_{2}, \ldots, g_{n}\right)$.

Lemma 2.4. The maps $d_{i}, s_{j}$ defined on the spaces $E_{n}(q, G)$ and $B_{n}(q, G)$ are well-defined and equip them with the structure of simplicial spaces.

Proof. The maps $d_{i}$ and $s_{i}$ are well-defined since they are induced by group homomorphisms between free groups and from the fact that if $f: A \rightarrow B$ is a group homomorphism then $f\left(\Gamma^{q} A\right) \subseteq \Gamma^{q} B$. The simplicial identities follow (as in the classical case for the bar construction) from the definition of the homomorphisms inducing the maps $d_{i}$ and $s_{j}$.

Note that the map $G^{n+1} \rightarrow G^{n}$ that projects the last $n$ coordinates onto $G^{n}$ defines a simplicial map $p_{*}: E_{*}(q, G) \rightarrow B_{*}(q, G)$. Moreover, $G$ acts from the left on $E_{n}(q, G)$ by multiplication on the first coordinate $g\left(g_{0}, g_{1} \ldots, g_{n}\right)=\left(g g_{0}, g_{1}, \ldots, g_{n}\right)$ and this action makes $E_{*}(q, G)$ into a $G$-simplicial space; that is, the action of $G$ commutes with the face and degeneracy maps. This action is free and its degree-wise orbit space is homeomorphic to $B_{*}(q, G)$ [16]. Next recall the notion of geometric realization for a simplicial space [17].

Definition 2.5. The geometric realization of a simplicial space $Z_{*}$ is the following topological space $\left|Z_{*}\right|:=\coprod_{n>0} Z_{n} \times \Delta^{n} / \sim$, where $\Delta^{n}$ denotes the $n$-simplex and the equivalence relation $\sim$ is defined as follows. Identify $\left(x, \delta_{i} t\right) \in X_{n} \times \Delta^{n}$ with $\left(d_{i} x, t\right) \in X_{n-1} \times \Delta^{n-1}$ for any $x \in X_{n}, t \in \Delta^{n-1}$ and $\left(x, \sigma_{j} t\right) \in X_{n} \times \Delta^{n}$ with $\left(s_{j} x, t\right) \in X_{n+1} \times \Delta^{n+1}$ for any $x \in X_{n-1}$ and $t \in \Delta^{n+1}$. The topology on $\left|Z_{*}\right|$ is the quotient topology.

Definition 2.6. The realization $\left|Z_{*}\right|$ of a simplicial space has a canonical filtration defined as follows: $F_{r}\left|Z_{*}\right| \subset\left|Z_{*}\right|$ is the image of $\coprod_{0 \leq i \leq r} Z_{i} \times \Delta^{i}$ in the quotient space. The associated graded space is defined as $E_{j}^{0}\left(\left|Z_{*}\right|\right)=F_{j}\left|Z_{*}\right| / F_{j-1}\left|Z_{*}\right|$.

Example 2.7. In the case of Definitions 2.2 , and 2.3 with $q=\infty,\left|E_{*}(\infty, G)\right|=E G$, $\left|B_{*}(\infty, G)\right|=B G$ and $p$ is the standard map $E G \rightarrow B G$. In other words, this yields Milgram's model for the universal principal $G$-bundle $E G \rightarrow B G$ ([13], 25], and [5], page 49). 
Notice that the spaces $E_{*}(q, G)$ and $B_{*}(q, G)$ are simplicial subspaces of the bar construction of $G$.

Definition 2.8. The geometric realizations of $E_{*}(q, G)$ and $B_{*}(q, G)$ are denoted by $E(q, G)$ and $B(q, G)$ respectively.

Note that the map $p: E(q, G) \rightarrow B(q, G)$ induced by $p_{*}$ is a principal $G$-bundle as it can be thought of as the natural pullback of the principal bundle $E G \rightarrow B G$ using the inclusion $B(q, G) \rightarrow B G$ (see [25], page 364, Theorem 8.3). The following definition will be used later and is useful for the analysis of the local structure of the map $p$ on the level of geometric realizations.

Definition 2.9. Let $S_{n}(j, q, G)$ be the subspace of $H o m\left(\mathcal{F}_{n} / \Gamma^{q}, G\right)$ obtained by taking $n$ tuples with at least $j$ coordinates equal to $1_{G}$.

Proposition 2.10. Let $G$ be a topological group.

1) The natural surjection $\mathcal{F}_{n} / \Gamma^{q+1} \rightarrow \mathcal{F}_{n} / \Gamma^{q}$ induces a map of simplicial spaces compatible with the simplicial maps $p_{n}$; that is, there is a commutative diagram

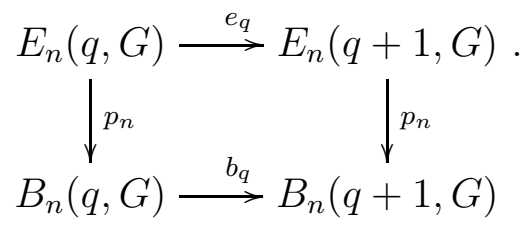

2) There are natural morphisms of principal G-bundles

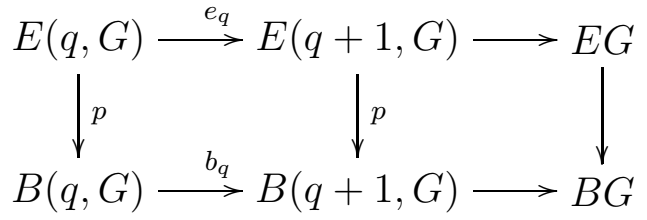

3) The maps $e_{q}, b_{q}$ yield a natural filtration of subspaces for $E G$ and $B G$.

4) If $G$ is a nilpotent group of class $c$, then $E(c+1, G)=E G, B(c+1, G)=B G$ and the filtration is finite. If $G$ is finitely generated, then the filtration is finite if and only if $G$ is nilpotent.

Proof. Part (1) and (2) are immediate since the maps $e_{q}, b_{q}$ are induced by group homomorphisms, whereas part (3) follows from (2). If $G$ is nilpotent of class $c$, then $\Gamma^{c+1}(G)=\{1\}$ and thus $\operatorname{Hom}\left(\mathcal{F}_{n} / \Gamma^{c+1}, G\right)=G^{n}$ for all $n$. Hence $E(c+1, G)=E G$ and $B(c+1, G)=B G$. If $B(q, G)=B G$ for some $q$, then for all $m$

$$
F_{m} B(q, G)-F_{m-1} B(q, G)=F_{m} B(\infty, G)-F_{m-1} B(\infty, G) .
$$

The relations imposed by the face and degeneracy maps show that

$$
F_{m} B(q, G)-F_{m-1} B(q, G)=\left(H o m\left(\mathcal{F}_{m} / \Gamma_{q}, G\right)-S_{m}(1, q, G)\right) \times\left(\Delta_{m}-\partial \Delta_{m}\right)
$$


so the formula above implies that

$$
\operatorname{Hom}\left(\mathcal{F}_{m} / \Gamma_{q}, G\right)-S_{m}(1, q, G)=\operatorname{Hom}\left(\mathcal{F}_{m}, G\right)-S_{m}(1, \infty, G) \text {. }
$$

Suppose that $G$ is generated by $m$ elements and that $\phi: \mathcal{F}_{m} \rightarrow G$ is a homomorphism onto the generators of $G$. Note that $\phi \in \operatorname{Hom}\left(\mathcal{F}_{m}, G\right)-S_{m}(1, \infty, G)$. Therefore $\Gamma^{q}(G)=$ $\phi\left(\Gamma^{q}\right)=\{1\}$ and $G$ is nilpotent.

\section{Further Properties of $\operatorname{Hom}\left(\mathcal{F}_{n} / \Gamma^{r}, G\right), E(q, G)$ And $B(q, G)$}

The functor from topological groups to topological spaces, given by $G \mapsto B(q, G)$ has a number of interesting features, some of them analogous to the classifying space functor but there are some important differences.

Proposition 3.1. The functor $B(q, G)$ satisfies the following properties:

(1) If $q \geq 2$ and $H$ is a topological group of nilpotency class less than $q$, then $B(q, H)=$ $B H$, the usual classifying space.

(2) If $G$ is a finite group then there exists an $N$ that depends on $G$ such that $B(q, G)=$ $B(N, G)$ for all $q \geq N$.

(3) If $q \geq 2$ and $\iota: H \rightarrow G$ is a homomorphism where $H$ is a group of nilpotency class less than $q$, then there is a commutative diagram

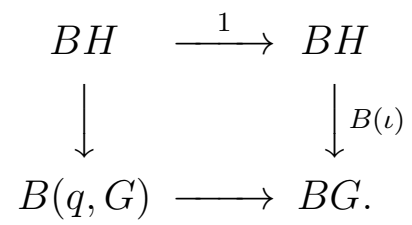

Thus if $B G$ is a stable retract of $B H$, then $B G$ is a stable retract of $B(q, G)$.

(4) If $q \geq 2$ and $Z$ is a central subgroup of $G$, then the multiplication map $\mu: Z \times G \rightarrow G$ induces an action $\mu: B Z \times B(q, G) \rightarrow B(q, G)$ together with a commutative diagram

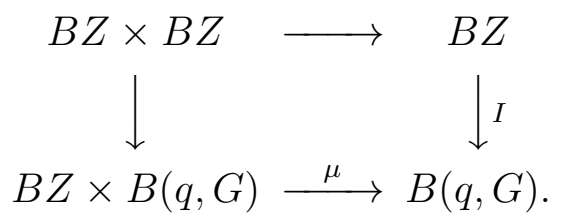

(5) The functor $B(q, G)$ commutes with direct limits in $G$, i.e. there are homeomorphisms

$$
B\left(q, \underset{\alpha}{\lim _{\alpha}} G_{\alpha}\right) \cong \underset{\alpha}{\lim } B\left(q, G_{\alpha}\right) .
$$

Proof. These properties are easy to verify, in particular the stable value $B(N, G)$ for the spaces $B(q, G)$ can be explained as follows. Let $N$ denote the minimal integer such that the class of nilpotence for any nilpotent subgroup of $G$ is strictly less than $N$. It suffices to show that $\operatorname{Hom}\left(\mathcal{F}_{n} / \Gamma^{N}, G\right)=\operatorname{Hom}\left(\mathcal{F}_{n} / \Gamma^{N+t}, G\right)$ for any $t \geq 1$. Given a homomorphism $\phi: \mathcal{F}_{n} \rightarrow G$, where $\mathcal{F}_{n}$ is a free group of rank $n$, let $H=\operatorname{Im} \phi$. Then $\phi$ will descend to a map $\mathcal{F}_{n} / \Gamma^{N+t} \rightarrow G$ if and only if the nilpotence class of $H$ is less than $N+t$. However, given that $H$ is nilpotent, its nilpotence class is in fact less than $N$; hence $\phi$ represents a unique 
element in $\operatorname{Hom}\left(\mathcal{F}_{n} / \Gamma^{N}, G\right)$ and the proof is complete. Note that if $G$ is nilpotent then the stable value of $B(q, G)$ is $B G$.

Recall that the map $c_{g}: B G \rightarrow B G$ induced by conjugation by $g \in G$ is homotopic to the identity. It turns out that for $B(q, G)$ this need not be the case. The reason is that $B_{*}(q, G)$ splits into a lattice of nerves of categories so that conjugation may permute this lattice. On the other hand, this difference indicates that when comparing the cohomology of $B G$ with that of $B(q, G)$ it is only necessary to look at the invariant part of the cohomology of $B(q, G)$ as the following diagram

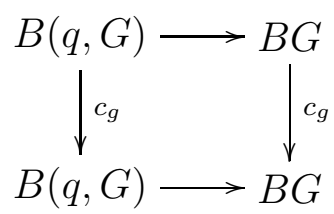

commutes. Hence the map $H^{*}(B G ; \mathbb{Z}) \rightarrow H^{*}(B(q, G) ; \mathbb{Z})$ factors through $H^{*}(B(q, G) ; \mathbb{Z})^{G}$.

The next proposition deals with basic facts about the cohomology of the spaces $B(q, G)$. These have close connections to properties of ordinary group cohomology.

Proposition 3.2. The functors $B(q, G)$ satisfy the following properties:

(1) If $G$ is a finite group with mod $p$ cohomology detected by subgroups of nilpotence class less than $q$, then the map induced on mod $p$ cohomology

$$
H^{*}\left(B G ; \mathbb{F}_{p}\right) \rightarrow H^{*}\left(B(q, G) ; \mathbb{F}_{p}\right)
$$

is a monomorphism.

(2) If $G$ is a finite group, then for any $q \geq 2$, the map induced on mod-p cohomology

$$
H^{*}\left(B G ; \mathbb{F}_{p}\right) \rightarrow H^{*}\left(B(q, G) ; \mathbb{F}_{p}\right)
$$

is a monomorphism modulo nilpotent elements.

(3) The spectral sequence obtained from the skeletal filtration satisfies

$$
E_{s, t}^{1}=\oplus H_{s+t}\left(F_{s} B(q, G), F_{s-1} B(q, G) ; \mathbb{Z}\right)
$$

and abuts to $H_{s+t}(B(q, G) ; \mathbb{Z})$. In case $q=\infty$, and coefficients are in a field $\mathbb{F}$, this spectral sequence is the bar spectral sequence with $E_{s, t}^{2}=\operatorname{Tor}_{s, t}{ }^{H_{*}}(G)(\mathbb{F}, \mathbb{F})$ which abuts to $H_{s+t}(B G ; \mathbb{F})$.

Proof. The first statement follows immediately from parts (1) and (2) of the previous proposition. For the second statement it suffices to prove it for $q=2$. Recall the result due to Quillen and Venkov, namely that if $A_{p}(G)$ denotes the poset of non-trivial elementary abelian $p$-subgroups of $G$ with morphisms induced by inclusion and conjugation, then the map induced by the restrictions $Q: H^{*}\left(B G ; \mathbb{F}_{p}\right) \rightarrow \lim _{E \in A_{p}(G)} H^{*}\left(E ; \mathbb{F}_{p}\right)$ has nilpotent kernel (see [20] and [5], page 146). Note, however, that this factors through the invariants of the $\bmod p$ cohomology of $B(q, G)$ for any $q \geq 2$; hence any element in the kernel of $\phi^{*}: H^{*}\left(B G ; \mathbb{F}_{p}\right) \rightarrow H^{*}\left(B(2, G) ; \mathbb{F}_{p}\right)$ must be nilpotent, as it is necessarily in the kernel of the map $Q$. 
The statement about the spectral sequence is routine and the identification with the bar spectral sequence in the classical case is well-known (see [15]).

Instead of considering the descending central series of a group, it is also possible to consider its $p$-descending central series, defined as follows:

Definition 3.3. Let $p$ denote a prime number and $Q$ a group; define a chain of subgroups $\Gamma_{p}^{r}(Q)$ inductively: $\Gamma_{p}^{1}(Q)=Q ; \Gamma_{p}^{i+1}(Q)=\left[\Gamma_{p}^{i}(Q), Q\right]\left(\Gamma_{p}^{i}(Q)\right)^{p}$. By convention, $\Gamma_{p}^{\infty}(Q)=\{1\}$. The $p$-descending central series of $Q$ is the normal series

$$
\cdots \subset \Gamma_{p}^{i+1}(Q) \subset \Gamma_{p}^{i}(Q) \subset \cdots \subset \Gamma_{p}^{2}(Q) \subset \Gamma_{p}^{1}(Q)=Q .
$$

A discrete group $Q$ is said to be $p$-nilpotent of there is some integer $m$ such that $\Gamma_{p}^{m+1}(Q)=$ $\{1\}$. The least such integer is called the $p$-class of $Q$. Given the free group $\mathcal{F}_{n}$ on $n$ letters, denote by $\Gamma_{p}^{q}$ the $q$-th stage of the descending central series for $\mathcal{F}_{n}$. There are some important differences here, in particular $\Gamma_{p}^{i} / \Gamma_{p}^{i+1}$ is an elementary abelian $p$-subgroup and the quotient groups $\mathcal{F}_{n} / \Gamma_{p}^{i}$ are all finite $p$-groups. Just as before, they can be assembled to yield simplicial spaces $E_{*}(q, G, p)$ and $B_{*}(q, G, p)$ where $G$ is a topological group. The basic fact used is that if $f: A \rightarrow B$ is a group homomorphism then $f\left(\Gamma_{p}^{i}(A)\right) \subset \Gamma_{p}^{i}(B)$. We can take their geometric realization and thus obtain spaces $E(q, G, p)$ and $B(q, G, p)$; they will now exhibit properties specific to the prime $p$. For example $B(2, G, p)$ is a space assembled from the $p$-elementary abelian subgroups in $G$. Here is an example of how these spaces capture $p$-local information.

Proposition 3.4. Let $G$ denote a finite group such that its mod p cohomology is detected on p-elementary abelian subgroups; then the map $B(2, G, p) \rightarrow B G$ induces an injective map in $\bmod p$ cohomology.

The proof follows from restricting to elementary abelian subgroups. From the inclusions

$$
B(2, G, p) \subset B(2, G) \subset B G
$$

it follows that detecting on $B(2, G, p)$ is sharper than doing it with $B(2, G)$.

Example 3.5. Let $G=\Sigma_{n}$, the symmetric group on $n$ letters. Then it is well known (see [5], Ch. VI) that its mod 2 cohomology is detected on elementary abelian 2-groups. Thus it follows that for all $n \geq 1 H^{*}\left(\Sigma_{n} ; \mathbb{F}_{2}\right) \hookrightarrow H^{*}\left(B\left(2, \Sigma_{n}, 2\right) ; \mathbb{F}_{2}\right)$ is a monomorphism.

Remark. Other variations of these constructions can be obtained by using the free pro- $p$ groups or the profinite completions of the free groups. Likewise taking quotients under the natural conjugation action produces a simplicial space with terms of the form $\operatorname{Rep}\left(\mathbb{Z}^{n}, G\right)$. These constructions have interesting properties but will be considered elsewhere.

\section{Homotopy Properties of $B(q, G)$ for $G$ a Finite Group}

This section primarily addresses the case when $G$ is a finite group.

Definition 4.1. For a finite group $G$, let $\mathcal{N}_{q}(G)=\left\{A \subset G\right.$ subgroup $\left.\mid \Gamma^{\mathrm{q}}(\mathrm{A})=\{1\}\right\}$ 
The elements of $\mathcal{N}_{q}(G)$ are precisely all the subgroups of nilpotence class less than $q$. Note that this is a partially ordered set under inclusion, furthermore it is closed under conjugation by elements in $G$ and under the process of taking subgroups (indeed if $H \subset K$ is a subgroup, then $\Gamma^{i}(H) \subset \Gamma^{i}(K)$ for all $\left.i \geq 1\right)$. Such a collection is often referred to as a family of subgroups in $G$. A group can be defined associated to these subgroups and the inclusions between them.

Definition 4.2. If $G$ is a finite group, let $G(q)=\operatorname{colim}_{A \in \mathcal{N}_{q}(G)} A$.

Note that $\mathcal{N}_{2}(G)$ is the family of abelian subgroups in $G$ and $G(2)$ is simply the colimit of all abelian subgroups in $G$. The classifying spaces of the groups $G(q)$ will play a role in the analysis of the spaces $B(q, G)$.

Theorem 4.3. Let $G$ be a finite group, then for any $q \geq 2$,

$$
B(q, G)=\underset{A \in \mathcal{N}_{q}(G)}{\operatorname{colim}} B A .
$$

Proof. For any subgroup $H \subset G$, there are natural inclusions

$$
\operatorname{Hom}\left(\mathcal{F}_{n} / \Gamma^{q}, H\right) \subset \operatorname{Hom}\left(\mathcal{F}_{n} / \Gamma^{q}, G\right) .
$$

Given any homomorphism $\psi: \mathcal{F}_{n} \rightarrow G$ with $\psi\left(\Gamma^{q}\right)=1$, it follows that $K=\operatorname{Im} \psi$ belongs to $\mathcal{N}_{q}(G)$, and that $\psi \in \operatorname{Hom}\left(\mathcal{F}_{n} / \Gamma^{q}, \operatorname{Im} \psi\right)$. Now if $A \in \mathcal{N}_{q}(G)$, then $\operatorname{Hom}\left(\mathcal{F}_{n} / \Gamma^{q}, A\right)=$ $\operatorname{Hom}\left(\mathcal{F}_{n}, A\right)$ for all $n \geq 0$; combining these facts yields $B_{*}(q, G)=\bigcup_{A \in \mathcal{N}_{q}(G)} B_{*}(\infty, A)$. This simplicial space is contained in $B_{*}(\infty, G)$, and the realizations $B A$ are natural subspaces of $B G$. If $A \subset A^{\prime}$ then $B A \subset B A^{\prime}$; moreover $B A \cap B A^{\prime}=B\left(A \cap A^{\prime}\right)$. Therefore the realization $\left|\bigcup_{A \in \mathcal{N}_{q}(G)} B_{*}(\infty, A)\right|$ of the union is precisely the space that results from identifying the realizations $B A$ along their intersections i.e. the colimit, whence the result follows.

Note that the classifying space functor does not in general commute with colimits. In any case, since colimits are sometimes delicate to handle, it is preferable to work with a homotopy colimit whenever possible (see [28]). Under favorable circumstances they are weakly homotopy equivalent. This will occur if the diagram of spaces is a free diagram (see [9]); for a union this will hold if all the intersections of spaces in the diagram are used in the construction. This is automatically verified for the diagrams under consideration here, because of the nice properties of the functor $A \mapsto B A$ which have been outlined above.

Thus there is a weak equivalence $B(q, G) \simeq \underset{A \in \mathcal{N}_{q}(G)}{\operatorname{hocolim}} B A$. Now the identity element in $G$ makes all of the spaces in these diagrams pointed spaces (i.e. the classifying space $B\{1\}$ is the natural basepoint $*)$. From this it follows that the fundamental group of $B(q, G)$ can be computed as a colimit $\pi_{1}(B(q, G), *) \cong \operatorname{colim}_{A \in \mathcal{N}_{q}(G)} A \cong G(q)$. This can be expressed more succinctly as follows. Let $\left\{M_{i} \mid i \in I\right\}$ denote a collection of maximal subgroups in $\mathcal{N}_{q}(G)$. Then $\pi_{1}(B(q, G), *) \cong *_{i \in I} M_{i} /\left(r_{i j}\right)$ where the $r_{i j}$ are the relations coming from the inclusions $M_{i} \cap M_{j} \subset M_{i}$. 
Having established that $B(q, G)$ is a homotopy colimit, there are some well-known methods from homotopy theory that can be used to study its homotopy type.

Theorem 4.4. There is a natural fibration $B(q, G) \rightarrow B G(q)$ with fiber a simply-connected finite dimensional complex $K_{q}$.

Proof. If $A \in \mathcal{N}_{q}(G)$, then there is a natural inclusion $A \subset G(q)$. This gives rise to a fibration $G(q) / A \rightarrow B A \rightarrow B G(q)$, where the fiber is a discrete coset space. Taking homotopy colimits and applying the results in [19] (see [9], page 180) yields a fibration

$$
\underset{A \in \mathcal{N}_{q}(G)}{\operatorname{hocolim}} G(q) / A \rightarrow \underset{A \in \mathcal{N}_{q}(G)}{\operatorname{hocolim}} B A \rightarrow B G(q)
$$

and as the fiber $K_{q}$ is a homotopy colimit of a finite collection of discrete spaces it is necessarily finite dimensional. On the other hand $B G(q)$ is a $K(\pi, 1)$ and the map $B(q, G) \rightarrow$ $B G(q)$ induces an isomorphism on fundamental groups, whence the result follows.

A basic question is that of determining under what conditions the fibre $K_{q}$ is contractible. Question: If $G$ is a finite group, are the spaces $B(q, G)$ Eilenberg-Mac Lane spaces of type $K(G(q), 1)$ ?

For any $q \geq 2$, the group $G(q)$ admits a natural surjection onto $G$, as every element of $G$ is contained in an abelian subgroup, and these groups all belong to $\mathcal{N}_{q}(G)$ for $q \geq 2$. i.e. there is a group extension $1 \rightarrow T(q) \rightarrow G(q) \rightarrow G \rightarrow 1$. The kernel is torsion-free by construction, as every finite group in $G(q)$ necessarily embeds in $G$. Thus there are no exotic finite groups appearing in the colimit. For all $A \in \mathcal{N}_{q}(G)$ the group $T(q)$ acts freely on the coset space $G(q) / A$, with quotient precisely $G / A$. Applying homotopy colimits, the fibrations $G(q) / A \rightarrow G / A \rightarrow B T(q)$ give rise to a fibration $K_{q} \rightarrow \underset{A \in \mathcal{N}_{q}(G)}{\operatorname{hocolim}} G / A \rightarrow B T(q)$. On the other hand, as in the proof of Theorem 4.4, the fibrations $G / A \rightarrow B A \rightarrow B G$ give rise to fibrations hocolim $G / A \rightarrow B(q, G) \rightarrow B G$. Comparing this to the natural fibration $E(q, G) \rightarrow B(q, G) \rightarrow B G$ it can be seen that there is an equivalence $E(q, G) \simeq \operatorname{hocolim}_{A \in \mathcal{N}_{q}(G)} G / A$ and that $\pi_{1}(E(q, G)) \cong T(q)$, which is a torsion-free group. Note that the hocolim term is a finite complex. These spaces can be assembled into a commutative diagram of fibrations:

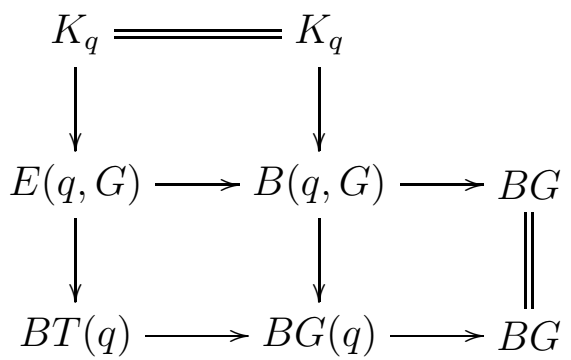

By the definition of $K_{q}$ in Theorem 4.4, $K_{q}$ is contractible if and only if $B(q, G) \simeq B G(q)$; this property is equivalent to $E(q, G) \simeq B T(q)$. 
The complexes $K_{q}$ are defined as homotopy colimits of functors with values in $G(q)$-spaces of the form $G(q) / A$ where $A$ is a finite group, and so admit a natural $G(q)$ action with finite stabilizers which restricts to a free action of the subgroup $T(q)$. The orbit space $K_{q} / T(q)$ is a finite complex with a natural $G$-action, and it is homotopy equivalent to $E(q, G)$. Now taking the quotient by $G(q)$ yields the equivalence $K_{q} / G(q) \simeq \operatorname{hocolim}_{A \in \mathcal{N}_{q}(G)}\{*\} \simeq\left|\mathcal{N}_{q}(G)\right| \simeq\{*\}$ due to the fact that the nerve of the category is contractible, as it has a minimal element (the trivial subgroup $\{1\})$. On the other hand, there is an identification $B(q, G) \simeq E G(q) \times_{G(q)} K_{q}$ which arises from the equivalence

$$
\underset{A \in \mathcal{N}_{q}(G)}{\operatorname{hocolim}} E G(q) \times_{G(q)} G(q) / A \simeq E G(q) \times_{G(q)} \underset{A \in \mathcal{N}_{q}(G)}{\operatorname{hocolim}} G(q) / A .
$$

Note that the projection map $B(q, G) \rightarrow\left|\mathcal{N}_{q}(G)\right|$ is a rational equivalence (as the isotropy is finite) and hence it follows that $H^{i}(B(q, G) ; \mathbb{Q}) \cong 0$ for $i>0$ (this also follows from Theorem 4.3 ).

Definition 4.5. Let $G$ denote a finite group and let $\mathcal{P}_{q}(G)$ be the category with objects the set $\left\{M_{\alpha}, M_{\alpha} \cap M_{\beta} \mid M_{\alpha}, M_{\beta} \in \mathcal{M}_{q}(G)\right\}$ where $\mathcal{M}_{q}(G)$ denotes the set of maximal subgroups in $G$ of nilpotence class $<q$, and the morphisms are the set of inclusions of the form $M_{\alpha} \cap M_{\beta} \rightarrow M_{\alpha}$.

This category is 1-dimensional, as there are no compositions. This can be identified with a graph of groups, that is, an oriented graph with a group at each vertex and a homomorphism between the two vertices of each edge according to orientation. Note that in this situation the graph is always connected.

Theorem 4.6. Let $q \geq 2$ and $G$ a finite group such that $\mathcal{P}_{q}(G)$ is a tree. Then the space $B(q, G)$ is aspherical and there is a natural homotopy equivalence $B(q, G) \simeq B G(q)$.

Proof. Using the graph of groups $\mathcal{P}_{q}(G)$ a space $\mathbb{B} \mathcal{P}(\mathcal{G})$ can be constructed by inserting a copy of the classifying space of each group at each vertex of the graph and by filling in a mapping cylinder for each map induced on classifying spaces on each edge (in this case arising from subgroup inclusions). The two ends of the mapping cylinder are identified with the classifying spaces on the respective vertices. This is a special case of a general construction for graphs of groups; in fact by [11], Theorem 1B.11 it follows that $\mathbb{B P}_{q}(G)$ is aspherical (the relevant hypothesis is the injectivity of all the homomorphisms on edges).

By construction the maps between classifying spaces have been replaced by cofibrations. Given that $\mathcal{P}_{q}(G)$ is a tree, the space $\mathbb{B P}_{q}(G)$ is homotopy equivalent to their colimit (by collapsing the mapping cylinders) and this is precisely $B(q, G)$.

It is interesting to note that in the situation above, the colimit group $G(q)$ can be readily understood using the theory of trees as in [22]; the special condition can be described as those which determine a tree of groups, and $G(q)$ is the corresponding inductive limit associated to the tree. 
Example 4.7. For the symmetric group $\Sigma_{4}$ the diagram of abelian subgroups is quite intricate although it can be shown that in fact $B\left(2, \Sigma_{4}\right)$ is aspherical. On the other hand the maximal subgroups of class less than 3 in $\Sigma_{4}$ are isomorphic to either $D_{8}$ or $\mathbb{Z} / 3$, and every pair of distinct copies of $D_{8}$ intersect along the subgroup

$$
K=\{1,(12)(34),(13)(24),(23)(14)\} \cong \mathbb{Z} / 2 \times \mathbb{Z} / 2 .
$$

Furthermore, $\mathcal{N}_{q}\left(\Sigma_{4}\right)=\mathcal{N}_{3}\left(\Sigma_{4}\right)$ for all $q \geq 3$, hence it follows from the theorem above that

$$
B\left(q, \Sigma_{4}\right) \simeq \bigvee B \mathbb{Z} / 3 \vee B\left(*_{K}^{3} D_{8}\right) \simeq B \operatorname{colim}_{A \in \mathcal{N}_{q}\left(\Sigma_{4}\right)} A
$$

Remark. The spaces $B(q, G, p)$ can also be analyzed using the methods discussed in this section. In particular it is not hard to show that $B(q, G, p)=\underset{K \in \mathcal{S}_{p}(G)}{\operatorname{col}} B(q, K, p)$ where $\mathcal{S}_{p}(G)$ is the poset of $p$-subgroups in $G$. This in turn can be further decomposed using the the classifying spaces of $p$-subgroups which are of $p$-nilpotence class less than $q$.

\section{Stable Splittings and a Counting Formula for Homomorphisms}

A stable splitting was given for the space of commuting $n$-tuples in [3]. Analogous stable splittings arise for $\operatorname{Hom}\left(\mathcal{F}_{n} / \Gamma^{q}, G\right)$ from the fat wedge filtration of the product $G^{n}$ where the base-point of $G$ is $1_{G}$.

Definition 5.1. As before, let $S_{n}(j, q, G)$ denote the subspace consisting of elements in $\operatorname{Hom}\left(\mathcal{F}_{n} / \Gamma^{q}, G\right)$ with at least $j$ coordinates equal to $1_{G}$. Let $S_{n}(q, G)$ denote $S_{n}(1, q, G)$. A Lie group $G$ is said have cofibrantly filtered elements if the natural inclusions $I_{j}$ : $S_{n}(j, q, G) \rightarrow S_{n}(j-1, q, G)$ are cofibrations for all $n, q$ and $j$ for which both spaces are non-empty.

It would seem that many Lie groups $G$ should have cofibrantly filtered elements; it is plausible to conjecture that this holds if $G$ is a closed subgroup of $G L(n, \mathbb{C})$. Note that in [3] the weaker condition of having cofibrantly commuting elements (i.e. the special case $q=2$ ) was indeed verified for these groups. The following result describes the stable structure of the spaces of homomorphisms $\operatorname{Hom}\left(\mathcal{F}_{n} / \Gamma^{q}, G\right)$ in terms of more recognizable pieces.

Proposition 5.2. If $G$ has cofibrantly filtered elements, then there are homotopy equivalences

$$
\Sigma H \operatorname{Hom}\left(\mathcal{F}_{n} / \Gamma^{q}, G\right) \rightarrow \bigvee_{1 \leq k \leq n} \Sigma \bigvee \operatorname{Hom}\left(\mathcal{F}_{k} / \Gamma^{q}, G\right) / S_{k}(q, G)
$$

and the natural filtration quotients

$$
E_{k}^{0}(B(q, G))=F_{k} B(q, G) / F_{k-1} B(q, G)
$$

of the geometric realization $B(q, G)$ are stably homotopy equivalent to the summands

$$
\operatorname{Hom}\left(\mathcal{F}_{k} / \Gamma^{q}, G\right) / S_{k}(q, G) \text {. }
$$


This result is a special case of very general splitting for simplicial spaces $X_{*}$ which are proper and simplicially NDR. Details of this appear in [2]; the main work is to verify these conditions for the simplicial spaces $B_{*}(q, G)$.

In the special case when $G$ is a finite group, the spaces of homomorphisms are finite sets, and the cofibrantly filtered condition is easily verified. The decomposition above can be interpreted as a numerical formula. The following definition will be helpful for keeping track of the numbers which will appear in counting the cardinality of these spaces of homomorphisms.

Definition 5.3. Let $G$ be a finite group; the integer $\lambda_{n}(q, G)$ is defined as the cardinality of $\operatorname{Hom}\left(\mathcal{F}_{n} / \Gamma^{q}, G\right)$, and the integer $\mu_{k}(q, G)$ is defined as the rank of $H_{0}\left(E_{k}^{0}(B(q, G) ; \mathbb{Z})\right.$.

An immediate consequence of Theorem 5.2 is the formula

Corollary 5.4. If $G$ is a finite group, then

$$
\lambda_{n}(q, G)=1+\sum_{1 \leq k \leq n}\left(\begin{array}{l}
n \\
k
\end{array}\right) \mu_{k}(q, G) .
$$

The special case $q=2$ is especially interesting as the formula above provides information on the cardinality of the set of commuting elements in a finite group.

Example 5.5. If $A$ denotes a finite abelian group, then this formula reduces to

$$
|A|^{n}=1+\sum_{1 \leq k \leq n}\left(\begin{array}{l}
n \\
k
\end{array}\right)(|A|-1)^{k} .
$$

Example 5.6. Consider the case when $G$ is a finite transitively commutative group (see Section 8 for their properties) with trivial center. The space $B_{*}(2, G)$ is the one-point union of the simplicial spaces $B_{*} C_{G}\left(a_{i}\right), 1 \leq i \leq N$, where each $C_{G}\left(a_{i}\right)$ is a maximal abelian subgroup and so in this case $\mu_{k}(2, G)=\sum_{1 \leq i \leq N}\left(\left|C_{G}\left(a_{i}\right)\right|-1\right)^{k}$ and

$$
\lambda_{n}(2, G)=1+\sum_{1 \leq k \leq n}\left(\begin{array}{l}
n \\
k
\end{array}\right) \sum_{1 \leq i \leq N}\left(\left|C_{G}\left(a_{i}\right)\right|-1\right)^{k} .
$$

This applies to $G=A_{5}$, the alternating group; there are three isomorphism classes of centralizers: $\mathbb{Z} / 2 \times \mathbb{Z} / 2$ (five copies), $\mathbb{Z} / 3$ (ten copies) and $\mathbb{Z} / 5$ (six copies). This yields

$$
\lambda_{n}\left(2, A_{5}\right)=1+\sum_{1 \leq k \leq n}\left(\begin{array}{l}
n \\
k
\end{array}\right)\left[5 \cdot 3^{k}+10 \cdot 2^{k}+6 \cdot 4^{k}\right]
$$

\section{The Spaces $B(q, G)$ for Connected Lie Groups}

In this section basic properties of the spaces $B(q, G)$ will be analyzed for connected Lie groups, including a calculation of the rational cohomology of $B(2, G)$ when $G$ is compact.

For a compact, connected Lie group $G$, consider the map

$$
\phi_{n}: G / T \times T^{n} \rightarrow \operatorname{Hom}\left(\mathbb{Z}^{n}, G\right)
$$


given by

$$
\left(g T, t_{1}, \ldots, t_{n}\right) \mapsto\left(g t_{1} g^{-1}, \ldots, g t_{n} g^{-1}\right) .
$$

As described in $\S 2$, an element in $\operatorname{Hom}\left(\mathbb{Z}^{n}, G\right)$ is represented as an ordered $n$-tuple of commuting elements in $G$. Notice that the Weyl group $W(G)=N(T) / T$ acts freely on $G / T \times T^{n}$ by

$$
\left(g T, t_{1}, \ldots, t_{n}\right) \cdot w=\left(g w T, w^{-1} t_{1} w, \ldots, w^{-1} t_{n} w\right)
$$

and that the map $\phi_{n}$ is invariant under this action. This yields the following commutative diagram

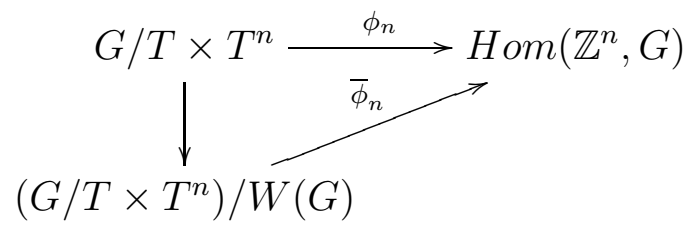

Note that the vertical map is equivalent to the map $\left(G \times T^{n}\right) / T \rightarrow\left(G \times T^{n}\right) / N(T)$. Assume that the space $\operatorname{Hom}\left(\mathbb{Z}^{n}, G\right)$ is path-connected, then as was shown in [6], $\phi_{n}$ is surjective and the fibers of the induced map $\overline{\phi_{n}}:\left(G \times T^{n}\right) / N(T) \rightarrow \operatorname{Hom}\left(\mathbb{Z}^{n}, G\right)$ are rationally acyclic. Hence applying the Vietoris-Begle Theorem it follows that $\overline{\phi_{n}}$ induces a rational homology equivalence and thus the map $\phi_{n}$ induces an isomorphism $H^{*}\left(\operatorname{Hom}\left(\mathbb{Z}^{n}, G\right) ; \mathbb{Q}\right) \rightarrow$ $H^{*}\left(G / T \times T^{n} ; \mathbb{Q}\right)^{W(G)}$. This information can be assembled to yield the following theorem.

Theorem 6.1. Let $G$ denote a compact, connected Lie group with maximal torus $T \subset G$. Assume that the spaces $\operatorname{Hom}\left(\mathbb{Z}^{n}, G\right)$ are all path connected. There is an isomorphism

$$
H^{*}(B(2, G) ; \mathbb{Q}) \cong H^{*}(G / T \times B T ; \mathbb{Q})^{W(G)}
$$

which is compatible with the well-known isomorphism $H^{*}(B G ; \mathbb{Q}) \cong H^{*}(B T ; \mathbb{Q})^{W(G)}$, where $W(G)$ denotes the Weyl group of $T$ in $G$.

Proof. Identify the space $G / T \times T^{n}$ with the product $G / T \times B_{n}(\infty, T)$, where $G / T$ is seen as a constant simplicial space. Thus the maps $\phi_{n}$ define a simplicial map $\phi$, the Weyl group $W(G)$ acts simplicially on $G / T \times B_{*}(\infty, T)$ and $\phi$ is invariant under this action. This yields the following commutative diagram of simplicial spaces

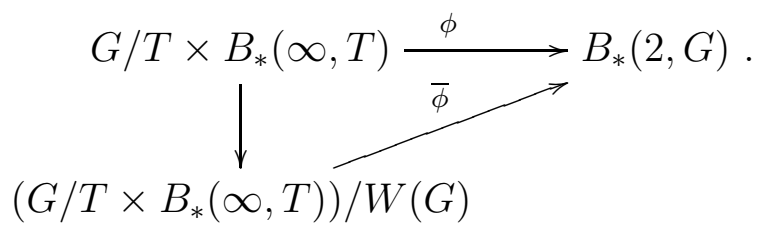

The simplicial spaces under consideration are all proper (note that this was proved in [3] for $B_{*}(2, G)$ ) and so by a standard result (see [14]) the rational cohomology isomorphisms induced by the maps $\bar{\phi}_{n}$ induce an isomorphism between the rational cohomology of the 
geometric realizations, yielding the desired equivalence. The last assertion follows from the diagram

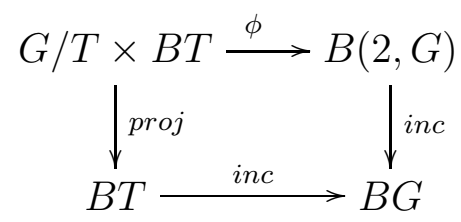

which is commutative up to homotopy.

Remark. If the spaces $\operatorname{Hom}\left(\mathbb{Z}^{n}, G\right)$ are not path-connected, an analogous result can be obtained using the generic component $\operatorname{Hom}_{0}\left(\mathbb{Z}^{n}, G\right)$ i.e. the component of the trivial representation. This is indeed a simplicial subspace, as the face and degeneracy maps are continuous and preserve the trivial representation. Its realization gives rise to a subspace $B(2, G)_{0} \subset B(2, G)$ for which the rational cohomology can be computed as above.

Example 6.2. In case $G=U(n)$, it was shown in [3] that the spaces of ordered commuting $n$-tuples are all path-connected. Hence the theorem applies here. Note that in this case the Weyl group is the symmetric group $S_{n}$ which acts by permuting the entries in the diagonal maximal torus. Recall that $H^{*}(B T) \cong \mathbb{Q}\left[t_{1}, \ldots, t_{n}\right]$, a polynomial ring on $n$ two dimensional generators. Thus the rational cohomology of $B(2, U(n))$ can be described as the ring of invariants $H^{*}(B(2, U(n)) ; \mathbb{Q}) \cong\left(H^{*}(U(n) / T) \otimes \mathbb{Q}\left[t_{1}, \ldots, t_{n}\right]\right)^{S_{n}}$. Note that the natural inclusion $B(2, U(n)) \rightarrow B U(n)$ has a stable section, as this is true for the map $B T \rightarrow B U(n)$, where $T \subset U(n)$ is a maximal torus [23]. Thus $B U(n)$ is a stable retract of $B(q, U(n))$ for all $q \geq 2$.

Remark. The spaces $\operatorname{Hom}\left(\mathbb{Z}^{n}, G\right)$ admit an action of $G$ by conjugation, and the orbit space $\operatorname{Rep}\left(\mathbb{Z}^{n}, G\right)$ can be identified with the moduli space of flat $G$-bundles over the torus $T^{n}$, an object of considerable geometric interest. The structure of these spaces in the case of $G=U(n), S U(n), S p(n)$ is given in [4].

The next result in this section is a product decomposition arising from looping the fibration $E(q, G) \rightarrow B(q, G) \rightarrow B G$. First recall that there is a map $\iota_{G}: \Sigma(G) \rightarrow B G$ which is natural for morphisms of topological groups obtained by identifying the first filtration $F_{1} B G$ as $\Sigma(G)$. In addition, the composite

$$
G \stackrel{E}{\longrightarrow} \Omega \Sigma(G) \stackrel{\Omega\left(\iota_{G}\right)}{\longrightarrow} \Omega B G
$$

is a homotopy equivalence where $E$ is the standard Freudenthal suspension map and $G$ is a Lie group.

Theorem 6.3. If $G$ is a connected Lie group, then the associated looped fibration

$$
\Omega(E(q, G)) \rightarrow \Omega(B(q, G)) \rightarrow \Omega(B G)
$$

has a cross-section (up to homotopy). The cross-section is induced by a map $\sigma(q, G): G \rightarrow$ $\Omega(B(q, G))$ given by the composite 


$$
G \stackrel{E}{\longrightarrow} \Omega(\Sigma(G)) \stackrel{\Omega(\iota(q, G))}{\longrightarrow} \Omega(B(q, G))
$$

where $\iota(q, G): \Sigma(G) \rightarrow B(q, G)$ is given by the canonical identification $\Sigma(G)=F_{1} B(q, G)$ for $q \geq 2$, and which is natural for morphisms in $G$. Thus there is a homotopy equivalence $\theta(q, G): G \times \Omega(E(q, G)) \rightarrow \Omega B(q, G)$.

Furthermore the cross-section $\sigma(q, G)$ and homotopy equivalence $\theta(q, G)$ are natural in the sense that if $f: G \rightarrow H$ is a morphism of topological groups, then there are strictly commutative diagrams

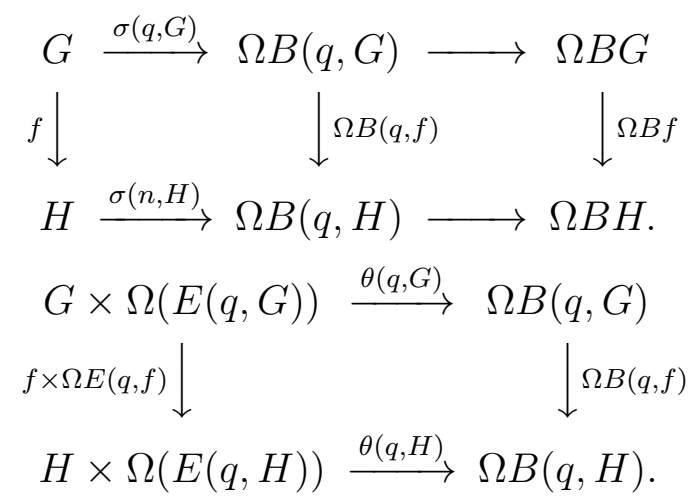

Proof. Recall that there is a canonical identification $\Sigma(G)=F_{1} B(q, G)$ for $q \geq 2$ natural for morphisms in $G$. Thus there is a natural factorization

$$
\Sigma(G) \longrightarrow F_{1} B(2, G) \longrightarrow \cdots \longrightarrow F_{1}(B G) .
$$

together with the associated composite arising by taking adjoints

$$
G \stackrel{E}{\longrightarrow} \Omega \Sigma(G) \longrightarrow \Omega F_{1} B(2, G) \longrightarrow \cdots \longrightarrow \Omega\left(F_{1}(B G)\right) \longrightarrow \Omega(B G) .
$$

So the following diagram strictly commutes:

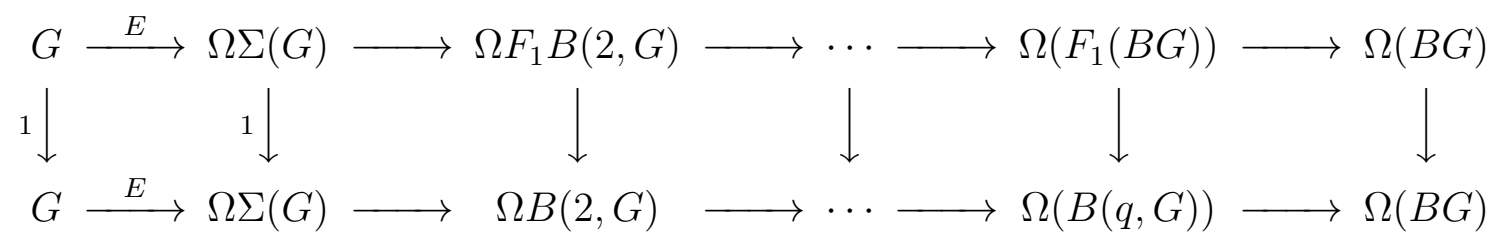

The composite

$$
G \stackrel{E}{\longrightarrow} \Omega \Sigma(G) \longrightarrow \Omega B(2, G) \longrightarrow \cdots \longrightarrow \Omega(q, G))
$$

is the adjoint $\iota(q, G): \Sigma(G) \rightarrow B(q, G)$ and thus the induced map

$$
G \stackrel{E}{\longrightarrow} \Omega \Sigma(G) \longrightarrow \Omega(B(q, G)) \longrightarrow \Omega(B G)
$$

is a homotopy equivalence. 
The looped fibration $\Omega(E(q, G)) \rightarrow \Omega(B(q, G)) \rightarrow \Omega(B G)$ has a cross-section. The choice of homotopy equivalence $\theta(q, G): G \times \Omega(E(q, G)) \rightarrow \Omega(B(q, G))$ is the composite

$$
G \times \Omega(E(q, G)) \stackrel{\sigma(q, G) \times \Omega(p(q, G))}{\longrightarrow} \Omega(B(q, G)) \times \Omega(B(q, G)) \stackrel{\text { multiply }}{\longrightarrow} \Omega(B(q, G)) .
$$

Thus the total space $\Omega B(q, G)$ is homotopy equivalent to a product $G \times \Omega(E(q, G))$.

To finish, it suffices to check naturality. Notice that the maps $\sigma(q, G): G \rightarrow \Omega B(q, G)$ are natural for morphisms in $G$; the maps $\Omega(p(q, G)): \Omega(E(q, G)) \rightarrow \Omega(B(q, G))$ are natural for morphisms in $G$; and the composite

$$
G \times \Omega(E(q, G)) \stackrel{\sigma(q, G) \times \Omega(p(q, G))}{\longrightarrow} \Omega(B(q, G)) \times \Omega(B(q, G)) \stackrel{\text { multiply }}{\longrightarrow} \Omega(B(q, G))
$$

is natural for morphisms in $G$. The theorem follows.

Remark. The proof of Theorem 6.3 does not require the hypothesis that $G$ be connected. Namely, the fibration $B(q, G) \rightarrow B G$ is, after looping, always split for any $G$ (as seen in the proof above). Since a multiplicative fibration which is split is a trivial fibration, there is a homotopy equivalence $G \times \Omega(E(q, G)) \simeq \Omega B(q, G)$. This decomposition does not usually preserve the loop structure as the following example shows. Consider the group $G=A_{5}$ : in this case, the space $\pi_{0} \Omega B\left(q, A_{5}\right)=\pi_{1}\left(B\left(2, A_{5}\right)\right)$. On the other hand, there is an isomorphism of groups

$$
\pi_{0}\left(A_{5} \times \Omega\left(E\left(2, A_{5}\right)\right)=A_{5} \times F\right.
$$

where $F$ is a finitely generated free group of rank 854. Thus $\pi_{0}\left(A_{5} \times \Omega\left(E\left(2, A_{5}\right)\right)\right.$ and $\pi_{0} \Omega B\left(q, A_{5}\right)$ fail to be isomorphic as groups, although they are isomorphic as sets.

\section{The Homology of $B(q, G)$ when $G$ is Finite, and the Feit-Thompson}

\section{THEOREM}

The purpose of this section is to consider homological properties of the spaces $E(q, G)$ and $B(q, G)$ when $G$ is finite. In particular the homology groups $H_{*}(E(q, G) ; \mathbb{Z})$ are natural $\mathbb{Z} G$-modules with potentially interesting properties.

If $K_{*}$ is a simplicial set, then define $\mathbb{Z} K_{*}$ as the free abelian group on the simplices of $K_{*}$. The face maps of $K_{*}$ define boundary maps by the equation

$$
\partial_{n}=\sum_{i=0}^{n}(-1)^{i} d_{i}: \mathbb{Z} K_{n} \rightarrow \mathbb{Z} K_{n-1}
$$

and so $\mathbb{Z} K_{*}$ becomes a chain complex. The crux of this construction are the isomorphisms $H_{*}\left(\mathbb{Z} K_{*}\right) \cong H_{*}\left(\operatorname{Sing}\left(\left|K_{*}\right|\right)\right) \cong H_{*}\left(\left|K_{*}\right|\right)$ where $\operatorname{Sing}\left(\left|K_{*}\right|\right)$ is the singular complex of $\left|K_{*}\right|$. Consider $\mathbb{Z} B_{*}(q, G)$, which can be thought of as a subcomplex of $\mathbb{Z} B_{*}(\infty, G)$; in fact $\mathbb{Z} B_{*}(q, G)=\bigcup_{H \in \mathcal{N}_{q}(G)} \mathbb{Z} B_{*}(\infty, H)$. 
Note that $\mathbb{Z} B_{0}(q, G)=\mathbb{Z}$ and $\mathbb{Z} B_{1}(q, G)=\mathbb{Z}[G]$ (the free abelian group on $G$ ), so the chain complex looks like

$$
\cdots \rightarrow \mathbb{Z} B_{2}(q, G) \stackrel{\partial_{2}}{\rightarrow} \mathbb{Z}[G] \stackrel{\partial_{1}}{\rightarrow} \mathbb{Z}
$$

where $\partial_{1}=0$ and $\partial_{2}(x, y)=y-x y+x$. Define for each $q \geq 2$ a subgroup of $\mathbb{Z}[G]$ by

$$
\left.I_{q}(G)=\langle y-x y+x| \Gamma^{q}(\langle x, y\rangle)=1, \text { with } x, y \in G\right\rangle
$$

Thus $I_{2}(G) \subseteq I_{3}(G) \subseteq \cdots \subseteq I_{\infty}(G)$, where $\Gamma^{q}=\{1\}$ when $q=\infty$. Then $H_{1}(B(q, G))=$ $\mathbb{Z}[G] / I_{q}(G)$ and so there is a sequence of surjective maps

$$
H_{1}(B(2, G)) \rightarrow H_{1}(B(3, G)) \rightarrow \cdots \rightarrow H_{1}(B G)
$$

Corollary 7.1. If $G$ is a finite group and $q \geq 2$, then the $H_{i}(B(q, G) ; \mathbb{Z})$ are finite abelian groups for all $i>0$, and their torsion only occurs at primes dividing the order of $G$.

Proof. As observed previously, $\mathbb{Z} B_{*}(q, G)$ can be thought of as the union of the chain complexes generated by the maximal subgroups of class $<q$. Therefore a Mayer-Vietoris spectral sequence can be used to compute $H_{*}(B(q, G) ; \mathbb{Z})$, involving the homology of finite subgroups of $G$; hence their reduced homology is annihilated by $|G|$. Thus the only torsion involved is at the primes dividing $|G|$.

Similarly one can consider $\mathbb{Z} E_{*}(q, G)$; in this case $\mathbb{Z} E_{0}(q, G)=\mathbb{Z}$, and the complex looks like

$$
\cdots \rightarrow \mathbb{Z} E_{2}(q, G) \stackrel{\partial_{2}}{\rightarrow} \mathbb{Z} E_{1}(q, G) \stackrel{\partial_{1}}{\rightarrow} \mathbb{Z}[G]
$$

where $\partial_{1}(a, x)=a x-a$ and $\partial_{2}(a, x, y)=(a x, y)-(a, x y)+(a, x)$. As the natural projection $E_{*}(q, G) \rightarrow B_{*}(q, G)$ is a simplicial map this yields a map of chain complexes and hence one of homology groups. Note that this map on $H_{1}$ takes the form

$$
H_{1}(E(q, G)) \rightarrow H_{1}(B G), \quad(z, x) \mapsto x
$$

at the chain level. As before, there is a sequence of surjective maps

$$
H_{1}(E(2, G)) \rightarrow H_{1}(E(3, G)) \rightarrow \cdots \rightarrow H_{1}(E G) .
$$

The following result shows that the first homology group contains interesting information.

As has been discussed previously, there is a principal $G$-bundle $E(q, G) \rightarrow B(q, G)$ which in the case of a discrete group gives rise to a $G$-covering. One question which arises is to analyze the structure of the exact sequence of fundamental groups $1 \rightarrow \pi_{1}(E(q, G)) \rightarrow$ $\pi_{1}(B(q, G)) \rightarrow G \rightarrow 1$. In particular, this sequence gives a natural representation $\rho: G \rightarrow$ $\operatorname{Out}\left(\pi_{1}(E(q, G))\right.$; a pertinent question here would be to ask for natural properties of this representation. Passing to homology, the Serre spectral sequence associated to the fibration $E(q, G) \rightarrow B(q, G) \rightarrow B G$ gives rise to an exact sequence connecting the low dimensional homology of these groups. Interestingly, this can be used to reformulate the Feit-Thompson Theorem, which states that every finite group of odd order is solvable. 
Proposition 7.2. The Feit-Thompson Theorem is equivalent to the following result: for $G$ a finite group of odd order, the homomorphism $H_{1}(E(2, G) ; \mathbb{Z}) \rightarrow H_{1}(B(2, G) ; \mathbb{Z})$ is not surjective.

Proof. Consider the fibration $E(2, G) \rightarrow B(2, G) \rightarrow B G$ and its associated 5-term exact sequence in homology; this yields an exact sequence of the form

$$
H_{2}(B(2, G) ; \mathbb{Z}) \rightarrow H_{2}(B G ; \mathbb{Z}) \rightarrow H_{1}(E(2, G) ; \mathbb{Z})_{G} \rightarrow H_{1}(B(2, G) ; \mathbb{Z}) \rightarrow G /[G, G] \rightarrow 0 .
$$

The Feit-Thompson Theorem says that every odd order group is solvable, which is equivalent to the condition that $G /[G, G] \neq 1$ for all $G$ of odd order. This is precisely equivalent to the failure of surjectivity for the map $H_{1}(E(2, G) ; \mathbb{Z}) \rightarrow H_{1}(B(2, G) ; \mathbb{Z})$.

As noted previously $T(q)$ is the fundamental group of $E(q, G)$, so $H_{1}(E(q, G) ; \mathbb{Z}) \cong$ $T(q) /[T(q), T(q)]$. In the sequel examples will be given where the geometry of the spaces $E(q, G)$ will be explicitly determined (especially in the case $q=2$ ) and the map on $H_{1}$ analyzed in some detail.

\section{8. $B(2, G)$ For Transitively Commutative Groups}

In this section the space $B(2, G)$ will be described for a very particular class of finite groups, the transitively commutative groups.

Definition 8.1. A transitively commutative 1 or TC group $G$ is one satisfying the following condition: given elements $g, h, k \in G-Z(G)$, if $[g, h]=1=[h, k]$, then $[g, k]=1$.

Note that the TC-groups are classified (see [21], page 519 and Theorem 9.3.12); examples include abelian groups, groups with an abelian normal subgroup of prime index, e.g. dihedral and generalized quaternion groups, $S L\left(2, \mathbb{F}_{2^{n}}\right)$, with $n \geq 2$, and all nonabelian groups of order $<24$. The following elementary lemmas describe their structure (proofs are left to the reader).

Lemma 8.2. Let $G$ be a nonabelian group; the following are all equivalent to $G$ being a $T C$ group:

a) If $g \notin Z(G)$, then $C(g)$ is abelian.

b) If $[g, h]=1$, then $C(g)=C(h)$ whenever $g, h \notin Z(G)$.

c) If $A, B \leq G$ and $Z(G)<C_{G}(A) \leq C_{G}(B)<G$, then $C_{G}(A)=C_{G}(B)$.

Lemma 8.3. Let $G$ be a TC group with trivial center.

a) The Sylow subgroups of $G$ are abelian and intersect trivially.

b) $\left\{C_{G}(x) \mid x \notin Z(G)\right\}$ is the family of maximal abelian subgroups of $G$.

c) The maximal abelian subgroups of $G$ intersect trivially.

d) If $H$ is a maximal abelian subgroup and $P \in \operatorname{Syl}_{p}(H)$, then $P \in \operatorname{Syl}_{p}(G)$.

\footnotetext{
${ }^{1}$ This definition appears in [18], page 415. A related notion, that of a centralizer abelian or CA group, refers to the situation when commutativity is transitive on all non-trivial elements in the group. Note that $\mathrm{TC}$ with trivial center is equivalent to non-abelian $\mathrm{CA}$.
} 
Now let $a_{1}, \ldots, a_{k} \in G-Z(G)$ be a set of representatives of their centralizers so that $G=\bigcup_{1 \leq i \leq k} C_{G}\left(a_{i}\right)$ and no smaller number of centralizers covers $G$. Note that Lemma 8.2 shows that the groups defining this union do not depend on the choice of representatives. This number $k$ is called the number of centralizers that cover $G$. Moreover, note that each $C\left(a_{i}\right)$ is a maximal abelian subgroup of $G$ and that distinct centralizers intersect along the center $Z(G)$. Applying Theorem 4.6 in this situation yields the following

Proposition 8.4. If $G$ is a $T C$ group, then $B(2, G) \simeq B G(2)$, where $G(2)$ is defined in 4.2 and as a consequence of 8.2 is the amalgamated product of the maximal abelian subgroups of $G$ along the center of $G$. In particular, $E(2, G)$ is a $K(\pi, 1)$ as well.

Corollary 8.5. If $G$ is a TC group with trivial center, then

$$
B(2, G) \simeq \bigvee_{1 \leq i \leq k}\left(\prod_{p \| C_{G}\left(a_{i}\right) \mid} B P\right)
$$

where $P \in \operatorname{Syl}_{p}(G)$.

Proof. $B_{*}(2, G)$ is the one-point union of the simplicial spaces $B_{*}\left(2, C_{G}\left(a_{i}\right)\right)$, and it's easy to see that $C_{G}\left(a_{i}\right) \cong \prod_{p \| C_{G}\left(a_{i}\right) \mid} P$ with $P \in S y l_{p}(G)$. The result follows.

Proposition 8.6. If $G$ is a finite TC group with trivial center, then there is a stable homotopy equivalence $B(2, G) \simeq \bigvee_{p \| G \mid} \bigvee_{P \in S y l_{p}(G)} B P$.

Example 8.7. Let $G=S L_{2}\left(\mathbb{F}_{8}\right)$, this is a TC group of order $504=2^{3} \cdot 3^{2} \cdot 7$, with trivial center and with $p$-Sylow subgroups $(\mathbb{Z} / 2)^{3}, \mathbb{Z} / 9$ and $\mathbb{Z} / 7$. There is a stable homotopy equivalence

$$
B\left(2, S L_{2}\left(\mathbb{F}_{8}\right)\right) \simeq \bigvee^{9} B(\mathbb{Z} / 2)^{3} \bigvee\left[\bigvee^{28} B \mathbb{Z} / 9\right] \bigvee\left[\bigvee^{36} B \mathbb{Z} / 7\right]
$$

Consider the structure of $E(2, G)$ for $G$ a finite $T C$ group. The main ingredient which can be applied is the theory of trees, as described in [22]. In this situation, the colimit group $G(2)=*_{Z(G)} C_{G}\left(a_{i}\right)$ acts on a graph $X$ by setting

$$
\operatorname{Edges}(\mathrm{X})=\coprod_{\mathrm{k}}^{\mathrm{k}} \mathrm{G}(2) / \mathrm{Z}(\mathrm{G}), \quad \operatorname{Vertices}(\mathrm{X})=\mathrm{G}(2) / \mathrm{Z}(\mathrm{G}) \sqcup\left[\coprod_{1 \leq \mathrm{i} \leq \mathrm{k}} \mathrm{G}(2) / \mathrm{C}\left(\mathrm{a}_{\mathrm{i}}\right)\right]
$$

and where the $i^{\text {th }}$ copy of $G(2) / Z(G)$ is identified with the vertices $G(2) / Z(G) \sqcup G(2) / C\left(a_{i}\right)$ by using the identity and the natural maps $G(2) / Z(G) \rightarrow G(2) / C\left(a_{i}\right)$. It turns out that $X$ is a tree (see [22] p. 38), with a simplicial action of $G(2)$ having as fundamental domain the tree of groups given by

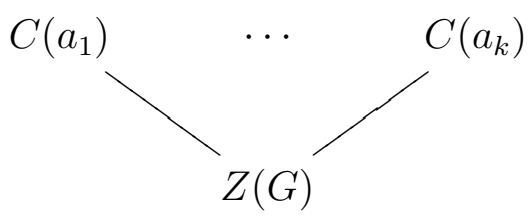


Proposition 8.8. If $G$ is a TC group then the fundamental group of $E(2, G)$ is a free group of rank

$$
N_{G}=1-|G: Z(G)|+\left(\sum_{1 \leq i \leq k}|G: Z(G)|-\left|G: C_{G}\left(a_{i}\right)\right|\right)
$$

and so $E(2, G) \simeq \bigvee_{N_{G}} S^{1}$.

Proof. Notice that the fundamental group of $E(2, G)$ is the kernel of the homomorphism between $G(2)$ to $G$ induced by the natural inclusions, and no element in $T(2)=\pi_{1}(E(2, G))$ is conjugate to an element in a centralizer or else the image of this element is nontrivial in $G$. Hence by a theorem due to Kurosh (see for instance Corollary A.2 from [8] or [22] p. 56) it must be a free group. To find the rank it suffices to calculate the Euler characteristic of the groups in the extension $1 \rightarrow T(2) \rightarrow G(2) \rightarrow G \rightarrow 1$ On the one hand $\chi(G(2))=\frac{\chi(T(2))}{|G|}$ and so rank $(\mathrm{T}(2))=1-\chi(\mathrm{T}(2))=1-\chi(\mathrm{G}(2))|\mathrm{G}|$. On the other hand, since $G(2)$ is the amalgamated product of the centralizers covering $G$ along $Z(G)$, it follows by an inductive argument that

$$
\chi(G(2))=\frac{1}{|Z(G)|}+\sum_{i=1}^{k}\left[\frac{1}{\left|C_{G}\left(a_{i}\right)\right|}-\frac{1}{|Z(G)|}\right]
$$

$$
\operatorname{rank} T(2)=1-\chi(G(2))|G|=1-|G|\left(\frac{1}{|Z(G)|}+\sum_{1 \leq i \leq k}\left[\frac{1}{\left|C_{G}\left(a_{i}\right)\right|}-\frac{1}{|Z(G)|}\right]\right)
$$

and the result follows.

Note that $Y=X / T(2)$ is a finite graph with a $G$-action. Looking at the cellular chain complex of $Y$ yields the following exact sequence of $\mathbb{Z} G$-modules

$$
0 \rightarrow H_{1}(E(2, G)) \rightarrow \bigoplus^{k} \mathbb{Z}[G / Z(G)] \stackrel{\phi}{\rightarrow} \mathbb{Z}[G / Z(G)] \oplus \bigoplus_{1 \leq i \leq k} \mathbb{Z}\left[G / C\left(a_{i}\right)\right] \rightarrow \mathbb{Z} \rightarrow 0
$$

where $\mathbb{Z}[G / H]$ denotes the usual permutation module with isotropy $H$, and the structure of $H_{1}(E(2, G))$ as a $G$-module is the one determined by the extension

$$
1 \rightarrow \pi_{1}(E(2, G)) \rightarrow G(2) \rightarrow G \rightarrow 1
$$

The map $\phi$ is described as follows; let $j_{i}: \mathbb{Z}[G / Z(G)] \rightarrow \mathbb{Z}\left[G / C\left(a_{i}\right)\right]$ be the natural projection induced by the inclusion of $Z(G)$ in $C\left(a_{i}\right)$ for $i=1, \ldots, k$. Then

$$
\phi\left(v_{1}, v_{2}, \ldots, v_{k}\right)=\left(\sum_{i=1}^{k} v_{i},-j_{1}\left(v_{1}\right), \ldots,-j_{k}\left(v_{k}\right)\right)
$$

There are two long exact sequences associated to this exact sequence, obtained by applying $G$-hypercohomology to it (see [5], Chapter V). The first one is:

$$
\cdots \rightarrow H_{i}(G ; \mathbb{Z}) \rightarrow H_{i-2}\left(G, H_{1}(E(2, G))\right) \rightarrow H_{i-1}(B(2, G) ; \mathbb{Z}) \rightarrow H_{i-1}(G ; \mathbb{Z}) \rightarrow \ldots
$$


The other one is of the form

$$
\cdots \rightarrow H_{q+1}(B(2, G)) \rightarrow \bigoplus H_{q}(Z(G)) \stackrel{\phi_{*}}{\rightarrow} H_{q}(Z(G)) \oplus\left[\bigoplus_{i=1}^{k} H_{q}\left(C\left(a_{i}\right)\right)\right] \rightarrow H_{q}(B(2, G)) \rightarrow \ldots
$$

If $A$ and $B$ are finite abelian groups and $A \subset B$, then $H_{*}(A) \subset H_{*}(B)$, whence it follows that $\phi_{*}$ is injective, and so for each $q \geq 0, H_{q}(B(2, G))$ can be described via the short exact sequence

$$
0 \rightarrow \bigoplus H_{q}(Z(G)) \stackrel{\phi_{*}}{\rightarrow} H_{q}(Z(G)) \oplus \bigoplus_{i=1}^{k} H_{q}\left(C\left(a_{i}\right)\right) \rightarrow H_{q}(B(2, G)) \rightarrow 0 .
$$

Now consider the special case when $G$ is a $T C$ group with a trivial center. In this situation the chain group $C_{1}(Y)$ is a free $\mathbb{Z} G$-module, and the $p$-Sylow subgroups of the centralizers $C_{G}\left(a_{i}\right)$ are $p$-Sylow subgroups for $G$.

Lemma 8.9. Let $M$ denote a permutation $\mathbb{Z} G$-module of finite rank and $\epsilon: M \rightarrow \mathbb{Z}$ the usual augmentation map onto the trivial module. Then the map $\epsilon$ splits over $\mathbb{Z} G$ if and only if $|G|$ divides the least common multiple of the orders of the isotropy subgroups.

Proof. We can write $M=\bigoplus_{i=1}^{k} \mathbb{Z}\left[G / H_{i}\right]$ where the $H_{i} \subset G$ are subgroups. If the map $\epsilon$ splits, then $\mathbb{Z} /|G|$ is a direct summand in $\widehat{H}^{0}(G, M) \cong \bigoplus_{i=1}^{k} \mathbb{Z} /\left|H_{i}\right|$ hence $|G|$ divides the l.c.m. of the orders of the subgroups $H_{1}, \ldots, H_{k}$. Conversely, suppose that $|G|$ divides this l.c.m. This means that it is possible to find a summand $\mathbb{Z} /|G| \subset \bigoplus_{i=1}^{k} \mathbb{Z} /\left|H_{i}\right|$ which maps bijectively onto $\widehat{H}^{0}(G ; \mathbb{Z})$ under the map induced in cohomology by $\epsilon$. Using the main result in [1], there exists a trivial submodule of rank one $T \subset M$ which represents this class of highest exponent and splits off as a direct summand of $M$; this defines the desired splitting.

Consider the case when $G$ is a TC group with trivial center. The kernel of the augmentation map $\epsilon: C_{0}(Y) \rightarrow \mathbb{Z}$ is isomorphic to the quotient module $C_{1}(Y) / H_{1}(E(2, G))$, where $C_{1}(Y)$ is a free $\mathbb{Z} G$ module. Applying the previous result yields a $G$-splitting: $C_{0}(Y) \cong \mathbb{Z} \oplus\left[C_{1}(Y) / H_{1}(E(2, G))\right]$. For the statement of the following corollary recall that given $M$ a $\mathbb{Z} G$-module, a finitely generated projective module $F$, and a surjection $f: F \rightarrow M$, then the kernel of $f$ is uniquely defined up to projective summands, and is denoted $\Omega^{1}(M)$ (see [5], Chapter II). Similarly if $N \rightarrow F^{\prime}$ is a monomorphism and $F^{\prime}$ is projective, then the cokernel is uniquely defined up to projective summands and denoted $\Omega^{-1}(N)$.

Corollary 8.10. If $G$ is a TC group with trivial center, then $H_{1}(E(2, G))$ is a $\mathbb{Z}$-torsion free module which up to projective factors is isomorphic to $\Omega^{1}(I)$, where $I$ denotes the kernel of the augmentation map $C_{0}(Y) \rightarrow \mathbb{Z}$. Furthermore up to projective summands there is a 
splitting of $\mathbb{Z} G-$ modules

$$
\mathbb{Z}[G / Z(G)] \oplus \bigoplus_{1 \leq i \leq k} \mathbb{Z}\left[G / C\left(a_{i}\right)\right] \cong \mathbb{Z} \oplus \Omega^{-1}\left(H_{1}(E(2, G))\right) .
$$

Example 8.11. $G=A_{5}$, the alternating group on five letters is a $T C$ group with trivial center. In this case $H_{1}\left(E\left(2, A_{5}\right)\right)$ is a module of rank equal to 854 and

$$
H_{i}\left(B\left(2, A_{5}\right)\right) \cong\left[H_{i}(\mathbb{Z} / 2 \times \mathbb{Z} / 2)\right]^{5} \oplus\left[H_{i}(\mathbb{Z} / 3)\right]^{10} \oplus\left[H_{i}(\mathbb{Z} / 5)\right]^{6}
$$

which in turn is isomorphic to $H_{i-1}\left(A_{5}, H_{1}\left(E\left(2, A_{5}\right)\right)\right) \oplus H_{i}\left(A_{5}\right)$.

Example 8.12. Let $G=Q_{8}$, the quaternion group of order eight. In this case the center is $\mathbb{Z} / 2$, and there are three maximal abelian subgroups (each of order four) intersecting along this central subgroup. In this case $B\left(2, Q_{8}\right) \cong B\left(*_{\mathbb{Z} / 2}^{3} \mathbb{Z} / 4\right)$, the amalgamation of the three groups along the common $\mathbb{Z} / 2$. The module $H_{1}\left(E\left(2, Q_{8}\right)\right)$ is of rank equal to three. It fits into an exact sequence

$$
0 \rightarrow H_{1}\left(E\left(2, Q_{8}\right)\right) \rightarrow \mathbb{Z}\left[Q_{8} / \mathbb{Z} / 2\right]^{3} \rightarrow \mathbb{Z}\left[Q_{8} / \mathbb{Z} / 2\right] \oplus \bigoplus_{i=1}^{3} Z\left[Q_{8} / C\left(a_{i}\right)\right] \rightarrow \mathbb{Z} \rightarrow 0
$$

and $H_{i}\left(B\left(2, Q_{8}\right)\right)$ can be computed as

$$
H_{i}\left(B\left(2, Q_{8}\right) ; \mathbb{Z}\right) \cong\left\{\begin{aligned}
\mathbb{Z} & \text { if } i=0 \\
\mathbb{Z} / 4 \oplus \mathbb{Z} / 2 \oplus \mathbb{Z} / 2 & \text { if } i>0 \text { odd } \\
0 & \text { if } i>0 \text { even }
\end{aligned}\right.
$$

Note that the sequence (I) also allows us to compute the character of the representation $G \rightarrow \operatorname{Aut}\left(H_{1}(E(2, G) \otimes \mathbb{C})\right.$, which will be denoted by $X_{E(2, G)}$. If $G$ is a TC group the kernel of the character $X_{E(2, G)}$ is precisely the center of $G$ and thus $G / Z(G) \rightarrow \operatorname{Aut}\left(H_{1}(E(2, G))\right.$ is a faithful representation. This result shows that the representation $G \rightarrow \operatorname{Out}\left(\pi_{1}(E(2, G))\right)$ is faithful when $G$ is a TC group with trivial center; moreover, the representation $\pi_{1}(B(2, G)) \rightarrow$ $\operatorname{Aut}\left(\pi_{1}(E(2, G))\right)$ is faithful as well.

\section{REFERENCES}

[1] A. Adem, Cohomological exponents of ZG-lattices, J. Pure Appl. Algebra 58(1989), no. 1, 1-5.

[2] A. Adem, M. Bendersky, A. Bahri. F. R. Cohen and S. Gitler, On decomposing suspensions of simplicial spaces, Bol. Soc. Mat. Mex. (3) Vol.15 (2009), 91-102.

[3] A. Adem, F. Cohen, Commuting Elements and Spaces of Homomorphisms, Mathematische Annalen 338 (2007), 587-626.

[4] A. Adem, F. Cohen, J. Gómez, Stable Spittings, Spaces of Representations and Almost Commuting Elements in Lie Groups, Math. Proc. Camb. Phil. Soc. Vol. 149 (2010), 455-490.

[5] A. Adem, R.J. Milgram, Cohomology of Finite Groups, Springer-Verlag Grundlehren 309 (2004).

[6] T. Baird, Cohomology of the space of commuting n-tuples in a compact Lie group, Algebr. Geom. Topol. 7 (2007), 737-754.

[7] V. Belyi, On Galois extensions of a maximal cyclotomic field, Math. USSR Izv., 14(1980), 247-256.

[8] K. Brown, Cohomology of Groups, Springer-Verlag GTM 87 (1982). 
[9] E. Dror-Farjoun, Cellular Spaces, Null Spaces and Homotopy Localization, Springer LNM $1622(1996)$.

[10] W. Feit, J. G. Thompson, Solvability of groups of odd order, Pacific J. Math. 13 (1963), 775-1029.

[11] A. Hatcher, Algebraic Topology, Cambridge University Press (2002).

$[12]$ Y. Ihara, On the embedding of $\operatorname{Gal}(\overline{\mathbb{Q}} / \mathbb{Q})$ in $\widehat{G T}$, in: The Grothendieck Theory of Dessins d'Enfants, London Mathematical Society Lecture Notes Vol. 200, Cambridge University Press, 1994.

[13] S. Mac Lane, The Milgram bar construction as a tensor product of functors, pp. 135-152, in Lecture Notes in Mathematics, Vol. 168 Springer, Berlin, 1970.

[14] J. P. May $E_{\infty}$ spaces, group completions, and permutative categories, New developments in topology (Proc. Sympos. Algebraic Topology, Oxford, 1972), pp. 61-93. London Math. Soc. Lecture Note Ser., No. 11, Cambridge Univ. Press, London, 1974.

[15] J. McCleary, A user's guide to spectral sequences, Cambridge Studies in Advanced Mathematics Vol. 58, Cambridge University Press, 2001.

[16] R. J. Milgram, The bar construction and abelian H-spaces, Ill. J. Math. 11 (1967), 242-250.

[17] J. Milnor, The geometrical realization of a semi-simplicial complex, Ann. of Math., 65 (1957), 357-362.

[18] J. Pakianathan and E. Yalcin, On commuting and noncommuting complexes, J. Algebra 236 (2001), 396-418.

[19] V. Puppe, A remark on homotopy fibrations, Manuscripta Math. 12 (1974), 113-120.

[20] D. Quillen, B.B. Venkov, Cohomology of finite groups and elementary abelian subgroups, Topology 11 (1972), 317-318.

[21] R. Schmidt, Subgroup lattices of groups, de Gruyter Expositions in Mathematics 14, Berlin, 1994.

[22] J.-P. Serre, Trees, Springer-Verlag, New York, 1980.

[23] V. Snaith, Algebraic cobordism and K-theory, Mem. Am. Math. Soc. 221 (1979).

[24] R. Solomon, A brief history of the classification of the finite simple groups, Bull. Amer. Math. Soc. (N.S.) 38 (2001), no. 3, 315-352.

[25] N. E. Steenrod, Milgram's classifying space of a topological group, Topology 7 (1968), 349-368.

[26] M. Suzuki, The nonexistence of a certain type of simple groups of odd order, Proc. Amer. Math. Soc. 8 (1957), 686-695.

[27] E. Torres-Giese, Spaces of Homomorphisms and Group Cohomology, Ph.D. Thesis, University of British Columbia (2007).

[28] V. Welker, G. Ziegler, R. Živaljević, Homotopy colimits-comparison lemmas for combinatorial applications, J. Reine Angew. Math., 509(1999), 117-149.

Department of Mathematics, University of British Columbia, Vancouver BC V6T 1Z2, CANADA

E-mail address: adem@math.ubc.ca

Department of Mathematics, University of Rochester, Rochester NY 14627, USA

E-mail address: cohf@math.rochester.edu

Department of Mathematics, University of Michigan, Ann Arbor Mi 48109, USA

E-mail address: etorresg@umich.edu 